\title{
Plasma Levels of Growth Arrest Specific Protein (Gas6) and the Soluble Form of Its Tyrosine Kinase Receptor Axl (sAxl) in Patients with Hepatocellular Carcinoma
}

\author{
Soichiro Uehara ${ }^{1}$, Katsuhiro Gotoh ${ }^{1}$, Hiroshi Handa ${ }^{1}$, Yoshiyuki Maki ${ }^{2}$ \\ ${ }^{1}$ Department of Internal Medicine and Gastroenterology, Hizirigaoka Hospital, Hunaoka, Japan; ${ }^{2}$ Primary Cell Co. Ltd., Sapporo, \\ Japan. \\ Email: ootaki_ph@ jikeikai.or.jp
}

Received February $22^{\text {nd }}, 2013$; revised March $24^{\text {th }}, 2013$; accepted March $31^{\text {st }}, 2013$

Copyright (C) 2013 Soichiro Uehara et al. This is an open access article distributed under the Creative Commons Attribution License, which permits unrestricted use, distribution, and reproduction in any medium, provided the original work is properly cited.

\begin{abstract}
The aim of this study was to determine the plasma levels of vitamin K-dependent protein growth arrest-specific protein 6 (Gas6) and its soluble receptor Axl (sAxl) in patients with hepatocellular carcinoma (HCC), acute hepatitis (AH), fulminant hepatitis(FH), chronic hepatitis $(\mathrm{CH})$, and liver cirrhosis (LC) and to determine whether Gas6 and sAxl can be used as biomarkers. Immunoassys were used to measure levels of plasma Gas6 and sAxl in 40 patients with HCC, 13 patients with $\mathrm{AH}, 3$ patients with $\mathrm{FH}, 7$ patients with $\mathrm{LC}$, and 20 healthy normal adult controls (NC). Furthermore, urinary $\gamma$-carboxyglutamic acid (Gla) was measured by high performance liquid chromatography. We have addressed this issue by conducting a cross-sectional study to determine whether plasma Gas6 and sAxl levels are associated with DCP, urinary $\gamma$-Gla, and liver functions in humans. Levels of Gas6, sAxl, and $\gamma$-Gla were significantly higher in HCC as compared to those in NC, and they were significantly positive correlated. Gas6/sAxl molar ratios in HCC were significantly higher than in NC, but those ratios in $\mathrm{AH}, \mathrm{FH}$, and LC were significantly lower in NC. Furthermore, Gas6/sAxl molar ratios in HCC increased significantly in comparison with those AH and LC. The increase of Gas6, sAxl and Gas6/sAxl molar ratio were correlated with the progression and poor prognosis of HCC. Thus, Gas6 and sAxl may be useful biomarkers for HCC.
\end{abstract}

Keywords: Gas6; Soluble Axl (sAxl); Receptor Tyrosine Kinase; $\gamma$-Carboxyglutamic Acid ( $\gamma$-Gla); Vitamin K; Hepatocellular Carcinoma (HCC)

\section{Introduction}

The growth arrest-specific gene gas6 (Gas6) is a number of vitamin $\mathrm{K}(\mathrm{VK})$-dependent ligand family, homologous to the protein S (PS), and is capable of binding to different cell surfaces via its $\mathrm{N}$ - and $\mathrm{C}$-termini, the $\mathrm{N}$-terminal Gla domain binding to phosphatidylserine-containing phospholipids and the C-terminal laminin G-like (LG) domain interacting with the TAM receptors, although the binding affinity of this ligand differs for each receptor (Axl > Sky > Mer), whereas PS has been shown to only Sky and Mer [1-3].

Gas6 is not primarily synthesized in the liver, but lung, kidney, intestine, ovary, testis, brain, bone marrow and vascular endothelium [3].

The Gas6/TAM system regulates multiple biological processes, including cell survival and proliferation, cell adhesion and migration, thrombus stabilization, and in- flammatory cytokine release. Therefore, the role of this system has been found to be important in inflammation, hemostasis, autoimmune disease, nervous, reproductive, and vascular systems and cancer [4].

In TAM-receptors, the Axl was originally found to be overexpressed in leukemia cells, but is expressed in various cancer cells. Increase in Axl expression is an indicator of poor prognosis for glioma [5] and kidney cancer [6]. Expression of Axl is also found in hepatoma cell lines $[7,8]$.

The membrane-bound Axl may be shedded from the cell membrane as a result of the activity of disintegrin-like metalloproteinase 10 (ADAM 10), and Axl is therefore present in circulation in a soluble form ( $\mathrm{sAxl})$ that consists of the extra cellular region of the protein. The levels of sAxl are normally in excess of Gas6 and that Gas6 is bound to sAxl in normal human blood [9]. 
On the other hand, vitamin $\mathrm{K}$ (VK) acts as a cofactor in the enzymatic carboxylation by $\gamma$-glutamylcarboxylase of glutamic acid in plasma proteins, and Gla residues formed from VK-dependent proteins cannot be reused and are excreted in urine. This quantitative measurement of urinary excretion of $\gamma$-Gla has shown to be reflective of total body pool of VK-dependent proteins, and these proteins turnover [10].

Des- $\gamma$-carboxyprothrombin (DCP) is known as a marker for hepapocellular carcinoma (HCC), and recent studies have revealed the usefulness of DCP as a diagnostic marker as well as its pivotal role in cancer progression [11]. In addition, DCP induces matrix metalloproteinase activity in HCC cells, thus promoting the migration and invasion of HCC cells in vitro [12].

Some case reports suggested that the possible protective effects of daily high-dose VK (menaquinone-4) treatment on the recurrence of HCC in successfully treated patients have been reported in several small trials. However, it is not necessarily decided about the evaluation $[13,14]$. In the present study, we paid attention to Gas6, which had TAM-system for the structure.

As a protocol, we measured Gas6 and sAxl mainly in the HCC patients and NC groups, and measured these in $\mathrm{AH}, \mathrm{FH}, \mathrm{CH}$, and $\mathrm{LC}$ patient groups secondarily.

Furthermore, we measured urinary $\gamma$-Gla as the marker of vitamin K metabolism and DCP in the same patient group. By analyzing these findings, we determined whether Gas6 and sAxl could become biomarkers as not only the diagnosis but also the effect of cancer therapy.

\section{Materials and Methods}

\subsection{Patients}

The study group comprised 40 consecutive patients with HCC (30 male, 10 female; $59.3 \pm 13.5$ years) treated at our hospital between 2008 and 2012, and 20 healthy normal adult controls (NC) matched for age (10 male, 10 females; mean age $57.8 \pm 18.5$ years), from whom informed consent was obtained. Furthermore, 33 cases of liver diseases including 13 cases of AH ( 9 males, 4 females; mean age $34.5 \pm 10.5$ years), 3 cases of FH (1 male, 2 females; mean ages 53.0 \pm 21.7 years), 5 cases of $\mathrm{CH}$ (2 male, 3 female; mean ages $58.8 \pm 7.4$ years), and 12 cases of LC ( 6 males, 6 females; mean ages $59.0 \pm 9.8$ years) were studied. All subjects gave written informed consent.

\subsection{Patients Background}

The diagnosis of HCC was performed by either imaging, ultrasonography, computed tomography, magnetic resonance imaging, angiography, and elevated serum con- centrations of $\alpha$-fetoprotein and DCP.

For the initial treatment of $40 \mathrm{HCC}$ cases, 10 (25\%), 3 (7.5\%), 19 (47.5\%), $5(12.5 \%)$ and $3(7.5 \%)$ received percutaneous ethanol injection (PEIT), radiofrequency ablation therapy (RFA), trans catheter arterial chemoembolization (TACE), interferon therapy, and symptomatic treatment, respectively. There were three survivals and 37 deaths to date. The cause of death is 25 cases by the disorder and the other disorders ware ten cases. The background liver abnormalities included chronic viral hepatitis in 17 cases $(42.5 \%)$, cirrhosis in 21 cases $(52.5 \%)$, and normal liver in 2 cases (5\%), associated with hepatitis B $(n=19)$, hepatitis C $(n=19)$. The pathological tumor node metastasis (TNM) stage of HCC according to the Liver Cancer Study Group of Japan $[15,16]$ was stage II in $4(10 \%)$, stage III in $14(35 \%)$, and stage IV in 22 $(55 \%)$.

The 13 cases of AH were all positive for IgM-anti HA antibody and cured within 8 weeks. The diagnosis of FH was performed in accordance with study of Fujiwara A., et al. $[17,18]$. Three cases fell into hepatic coma and hepatic failure within one week, and plasmapheresis treatment was performed in all cases, but led to death. The diagnosis of chronic hepatitis and LC was based on clinical and biochemical evidence, and confirmed by liver biopsy. Six patients were grade A, eight were graded B, and three were graded $\mathrm{C}$ under the Child-Pugh classification system. The 17 cases of $\mathrm{CH}$ and LC were hepatitis $\mathrm{B}$ $(\mathrm{n}=2)$, hepatitis $\mathrm{C}(\mathrm{n}=11)$, and normal $(\mathrm{n}=4)$. Blood samples from those patients were collected at days 7 of admission.

The etiology of the cases with liver abnormalities was determined by testing for HBsAg, HBV-DNA, IgM-antiHA (Dainabot, Tokyo, Japan), anti-HCV (Kokusai Siyaku, Kobe, Japan) and HCV-RNA (Roche Diagnotics, Mannheim, Germany).

Venous blood samples were taken on patients' admission. Plasma samples were collected in citric acid (3.8\%) and EDTA, and frozen immediately for storage at $-20^{\circ} \mathrm{C}$ until analysis.

The control group of liver and renal functions was within the normal ranges aspartate aminotransferase (AST), alanine aminotransferase (AST) $<40 \mathrm{IU} / \mathrm{L}$, bilirubin $<$ $0.8 \mathrm{mg} / \mathrm{dL}$, blood urea nitrogen $(\mathrm{BUN})<20 \mathrm{mg} / \mathrm{dL}$, creatinine $<1.0 \mathrm{mg} / \mathrm{dL}$ with no cases of anemia or hyperlipidemia (cholesterol $<200 \mathrm{mg} / \mathrm{dL}$ ).

\subsection{Determination of Gas6, and Soluble Axl (sAxl) Levels}

Measurement of Gas6, and sAxl levels were carried out using commercial ELISA kits that use the sandwich technique: DuoSet ${ }^{\circledR}$ (R\&D Systems, Minneapolis, MN, 
USA) for Gas6, and sAxl (Human Soluble Axl Elisa, Adipo Bioscience, Santa Clara, USA).

These intra- and inter-assay coefficients of variation for Gas6, and sAxl measurements were $6.4 \%$ and $7.9 \%$, and $6.7 \%$ and $8.0 \%$ respectively.

\subsection{Determination of DCP (PIVKA-II, and Urinary $\boldsymbol{\gamma}$-Gla levels}

Plasma levels of DCP were measured by using ELISA kit (Eitest PIVKA-IIkit; cut-off value, $40 \mathrm{mAU} / \mathrm{ml}$; Sanko Junyaku Co., Tokyo, Japan).

Urine samples were frozen at $-20^{\circ} \mathrm{C}$, subsequently thawed and centrifuged at $1500 \mathrm{~g}$ for $10 \mathrm{~min}$. The supernatant obtained was diluted with distilled water between 2 to 32 times, and the diluted sample was used for analysis. The Gla content of these samples was measured as described elsewhere Kuwada [19]. Urine samples were col- lected from 24-hour urine collections.

\subsection{Determination of Liver Enzymes, White Blood Cell Count, Platelet Counts, and Bilirubin}

Serum ALT, AST, bilirubin, and white blood cell and platelet counts were each measured by routine laboratory procedures. Furthermore, the etiology of the cases with hepatitis was determined by routine laboratory procedures for HBsAg, HBV-DNA, IgM-antiHA (Dainabot, Tokyo, Japan), anti-HCV (Kokusai Siyaku, Kobe, Japan) and HCV-RNA (Roche Diagnotics, Mannheim, Germany).

\subsection{Statistical Analysis}

Data are expressed as men \pm SD. Statistical analysis was carried out by using the Student' $t$-test and the level of significance was $\mathrm{P}<0.05$.

\section{Results}

\subsection{Plasma Levels of Gas6 in Cases with HCC, $\mathrm{AH}, \mathrm{FH}, \mathrm{CH}$, and $\mathrm{LC}$, and in Normal Adult Controls}

The plasma levels of Gas6 in cases with liver diseases and normal adult controls (NC) were shown in Table 1.

The plasma Gas6 levels in cases of HCC were significantly higher than in the healthy normal adults, and the levels in clinical HCC stages of II, III, and IV were increased significantly compared with normal adults.

Increase in Gas6 levels correlated with clinical stages of HCC. However, insignificant increase in levels of Gas6 were observed in patients with $\mathrm{AH}, \mathrm{FH}, \mathrm{CH}$ and LC as compared to those if NC (Table 1).

\subsection{Plasma Levels of $\mathbf{s A x l}$ in Cases of $\mathrm{HCC}, \mathbf{A H}$, FH, CH and LC, and in Normal Adults Controls}

The plasma levels of sAxl in cases of the liver diseases and NC were shown in Table 1.

The plasma levels of sAxl were significantly higher in cases with $\mathrm{HCC}, \mathrm{AH}, \mathrm{FH}$, and LC, than in the healthy $\mathrm{NC}$. The plasma sAxl levels in cases with $\mathrm{HCC}$ were higher than in the $\mathrm{NC}$, and increase in these levels was

Table 1. Plasma levels of Gas6, sAxl, Des $\boldsymbol{\gamma}$-carboxyprothrombin (DCP), and urinary levels of $\boldsymbol{\gamma}$-Gla in patients with hepatocellular carcinoma (HCC), acute hepatitis (AH), fulminant hepatitis (FH), chronic hepatitis (CH), liver cirrohsis (LC), and normal adult controls (NC).

\begin{tabular}{|c|c|c|c|c|c|c|}
\hline Diseases & & $\mathbf{n}$ & Gas6 (ng/ml) & sAxl (pg/ml) & DCP (AU/ml) & $\gamma$-Gla $(\mathbf{n M} / \mathrm{mg} \cdot \mathrm{Cr})$ \\
\hline Normal subjects (NC) & & 20 & $18.8 \pm 3.6$ & $271.8 \pm 19.2$ & $8.1 \pm 7.9$ & $68.9 \pm 17.9$ \\
\hline \multirow[t]{2}{*}{ Hepatocellular carcinoma (HCC) } & & 40 & $76.0 \pm 21.2^{* *}$ & $905.6 \pm 255.1^{* *}$ & $1582.8 \pm 1221.5^{* *}$ & $102.6 \pm 32.2^{*}$ \\
\hline & II & 4 & $56.3 \pm 22.4^{*}$ & $645.0 \pm 243.5^{*}$ & $252.3 \pm 166.9^{* *}$ & $70.1 \pm 36.0$ \\
\hline \multirow[t]{2}{*}{ HCC (TNM stage) } & III & 14 & $71.3 \pm 17.5^{* *}$ & $836.4 \pm 199.1^{* *}$ & $1126.1 \pm 1071.7^{* *}$ & $93.3 \pm 30.5^{*}$ \\
\hline & IV & 22 & $83.3 \pm 20.9^{* *+}$ & $997.5 \pm 250.9^{* *+}$ & $2081.8 \pm 1152.4^{* *}$ & $113.9 \pm 27.9^{* *+}$ \\
\hline Acute hepatitis $(\mathrm{AH})$ & & 13 & $25.0 \pm 10.7$ & $576.0 \pm 151.9^{* *}$ & $27.5 \pm 14.5^{* *}$ & $67.8 \pm 11.1$ \\
\hline Fulminant hepatitis $(\mathrm{FH})$ & & 3 & $53.0 \pm 7.0$ & $1246.0 \pm 418.0^{*}$ & $38.7 \pm 12.5^{*}$ & $59.0 \pm 15.9$ \\
\hline Chronic hepatitis $(\mathrm{CH})$ & & 5 & $16.0 \pm 4.8$ & $448.0 \pm 105.3$ & $23.1 \pm 9.7^{*}$ & $68.3 \pm 15.2$ \\
\hline Liver cirrhosis (LC) & & 13 & $27.9 \pm 12.7$ & $747.9 \pm 232.3^{* *}$ & $57.7 \pm 55.0^{* *}$ & $71.4 \pm 16.8$ \\
\hline
\end{tabular}

${ }^{*} \mathrm{P}<0.05,{ }^{* *} \mathrm{P}<0.01$ indicates statistical significance vs. NC. ${ }^{+} \mathrm{P}<0.05, \mathrm{HCC}$ II -stage indicates statistical significance vs. III,IV-stage. 
correlated with clinical stages of HCC. Significant difference in plasma levels of sAxl was also observed between clinical stages II, III, IV of HCC.

\subsection{Correlation between Plasma Levels of Gas6 and sAxl in Cases with HCC, AH, FH, CH and $\mathrm{LC}$}

A significant positive correlation was observed between Gas6 levels and sAxl levels in cases with all liver diseases. In particular, there was a significantly correlation between Gas6 and sAxl levels in cases with $\mathrm{HCC}(\mathrm{r}=$ $0.654, \mathrm{P}<0.01$; Figure 1).

Likewise, the coefficients of correlation between Gas6 and sAxl levels in $\mathrm{AH}, \mathrm{FH}, \mathrm{CH}$, and $\mathrm{LC}$ cases were 0.550 , $0.677,0.995,0.806$, respectively. These coefficients of correlation in cases of AH and LC were statistically significant.

\subsection{Plasma Levels of Gas6/sAxl Molar Ratio in Cases of HCC, AH, FH, CH, and LC, and Normal Adults Controls}

The Gas6 and sAxl levels in cases with the liver diseases were higher than in NC (Figure 1). The Gas6/ sAxl molar ratios in liver diseases were shown in Figure 2.

The median Gas6/sAxl molar ratios in NC, AH, FH, $\mathrm{CH}$, LC, HCC, HCC-II, HCC-III, and HCC-IV were 0.37 , $0.26,0.29,0.20,021,0.50,0.54,0.51$, and 0.50 respectively. Gas6/sAxl molar ratios in the TNM classification of HCC increased, however, the increased ratios

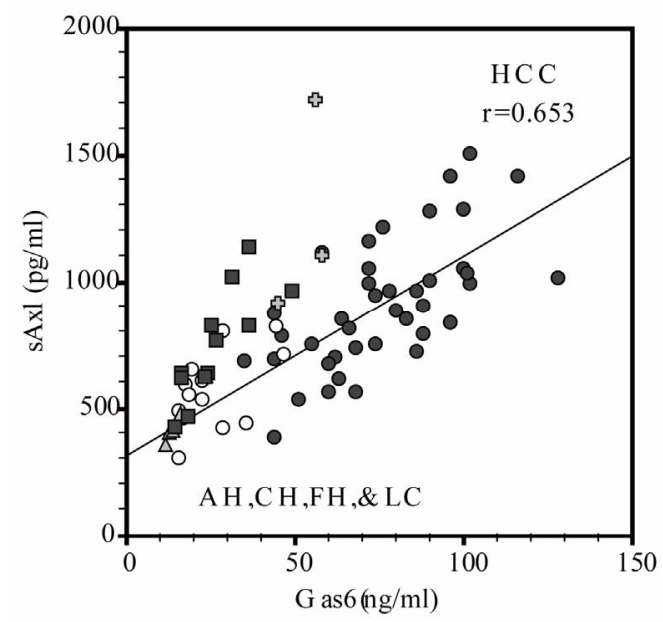

Figure 1. Correlation between Gas6 and sAxl in patients with hepatocellular carcinoma (HCC), acute hepatitis (AH), fulminant hepatitis (FH), chronic hepatitis (CH) and liver cirrhosis (LC). The coefficients of correlation between Gas6 and sAxl in HCC $(\bullet: \mathrm{r}=\mathbf{0 . 6 5 3}, \mathrm{n}=\mathbf{4 0}, \mathrm{P}<0.01)$, AH $(\mathrm{O}: \mathrm{r}=$ $0.550, n=13, P<0.05)$, FH ( $\mathrm{P}: \mathrm{r}=0.607, \mathrm{n}=3, \mathrm{P}<0.05)$, $\mathrm{CH}(\Delta: \mathrm{r}=0.995, \mathrm{n}=5, \mathrm{P}<0.05)$, and $\operatorname{LC}(\Delta: \mathrm{r}=0.806, \mathrm{n}=$ $12, \mathrm{P}<0.05)$, respectively. in II stage were not statistically significant, although the molar ratios in cases with $\mathrm{AH}, \mathrm{FH}$ and $\mathrm{LC}$ were lower as compared to those in normal adult controls. The Gas6/ sAxl molar ratios in cases with HCC increased significantly in comparison with those in cases with AH and LC (Figure 2).

\subsection{Correlation between Plasma Levels of Gas6 and DCP in Cases with HCC, AH, FH, CH, and $\mathrm{LC}$}

The coefficients of correlation between plasma Gas6 and DCP levels in cases of HCC, AH, FH, and LC were $0.135,-0.08,-0.999,-0.170$, and 0.485 respectively. Most of these correlations except FH were not statistically significant (Figure 3).

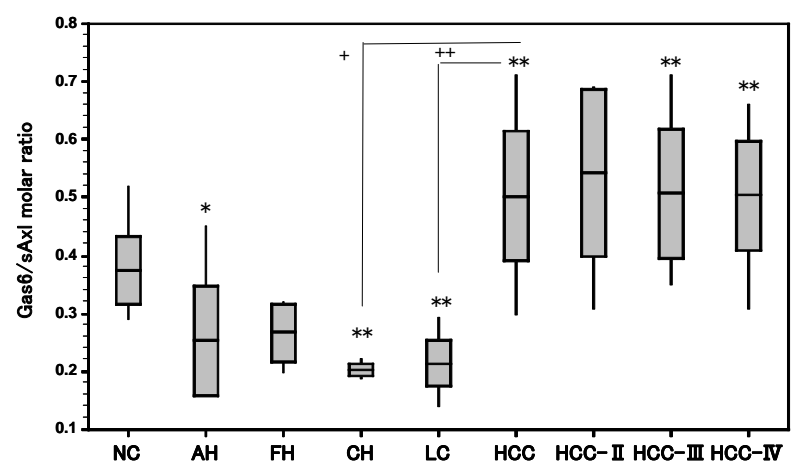

Figure 2. Gas6/sAxl molar ratios in patients with hepatocellular carcinoma (HCC), acute hepatitis(AH), fulminant hepatitis (FH), chronic hepatitis (CH), and liver cirrohsis (LC). ${ }^{*} \mathrm{P}<0.05,{ }^{* * *} \mathrm{P}<0.01$ vs. Normal controls (NC). ${ }^{+} \mathrm{P}<$ $0.05,{ }^{++} \mathrm{P}<0.01 \mathrm{CH}, \mathrm{LC}$ vs. HCC.

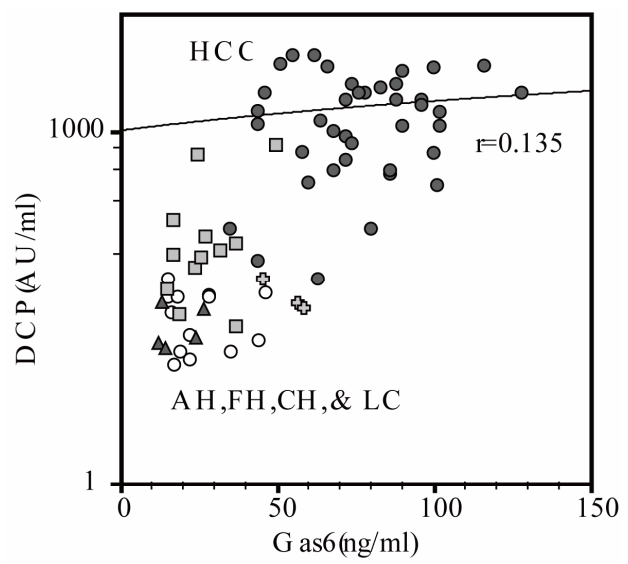

Figure 3. Correlation between Gas6 and DCP in patients with hepatocellular carcinoma (HCC), acute hepatitis (AH), fulminant hepatitis (FH), chronic hepatitis $(\mathrm{CH})$, and liver cirrhosis (LC). The coefficients of correlation between in HCC ( $\bullet: r=0.135, n=40)$, AH $(O: r=-0.081, n=13)$, FH $(+: \mathrm{r}=-0.991, \mathrm{n}=3, \mathrm{P}<0.05)$, and $\mathrm{CH}(\Delta: \mathrm{r}=-0.170, \mathrm{n}=$ 5), LC ( $\square: r=0.485, n=12)$, respectively. 


\subsection{Plasma Levels of DCP in Cases of $\mathrm{HCC}, \mathrm{AH}$, FH, CH and LC, and in Normal Adult Controls}

The plasma levels of DCP in cases of liver diseases and NC were shown in Table 1.

The plasma levels of DCP in cases of HCC were significantly higher than in healthy normal controls.

Furthermore, these values were elevated according to the TMN classification of the HCC and these statistical differences of the values between the stages were significant. Furthermore, positive correlation was seen between DCP and Gas6 levels in cases with HCC, but it was not significant correlation.

In both the cases of AH and FH, the DCP levels decreased than the cut/off values in HCC $(<40 \mathrm{mU} / \mathrm{ml})$, but the DCP levels in cases of LC slightly increased.

\subsection{Levels of Urinary $\gamma$-Gla Levels in Cases with HCC, AH, FH, and LC and in Healthy Normal Adult Controls}

The urinary $\gamma$-Gla in cases with HCC, AH, LC and in NC, were shown in Table 1. The urinary levels of $\gamma$-Gla increased significantly in cases with HCC were as compared to those in the NC. However, the urinary $\gamma$-Gla levels in cases with $\mathrm{AH}$, and $\mathrm{FH}$ were lower than in the $\mathrm{NC}$.

\subsection{Correlation between Plasma Gas6 Levels and Urinary $\gamma$-Gla Levels in Cases of HCC, $\mathrm{AH}, \mathrm{FH}$, and $\mathrm{LC}$}

The coefficients of correlation between levels of plasma Gas6 and urinary $\gamma$-Gla in patients with $\mathrm{HCC}, \mathrm{AH}, \mathrm{FH}$, $\mathrm{CH}$ and $\mathrm{LC}$ were $0.678,0.725,0.607,0.230$, and 0.674 respectively (Figure 4). Most of these correlations except CH were statistically significant (Figure 4).

\subsection{Correlation with Plasma Gas6, ALT, AST, Platelet Count, White Blood Cell Count, and Bilirubin in Cases of HCC, $\mathrm{AH}, \mathrm{FH}, \mathrm{CH}$ and LC}

The coefficients of correlation between Gas6, ALT, AST, platelet count, white blood cell count, and bilirubin, in cases of HCC $(\mathrm{n}=40)$ were $0.218,0.145,-0.105,0.202$, and 0.169 , respectively. In cases with $\mathrm{AH}(\mathrm{n}=13)$, those coefficients were $-0.068,-0.096,0.264,-0.277$, and -0.231 , respectively. In cases with FH $(\mathrm{n}=3)$, those coefficients were $-0.023,0.204,-0.099$, and 0.280 , and -0.887 , respectively. In cases with $\mathrm{CH}(\mathrm{n}=5)$, those coefficients were $0.422,0.425,0.036,0.249$, and 0.487 respectively. In cases with $\mathrm{LC}(\mathrm{n}=12)$, those coefficients were $0.422,0.501,0.274,-0.481$, and -0.278 respectively.

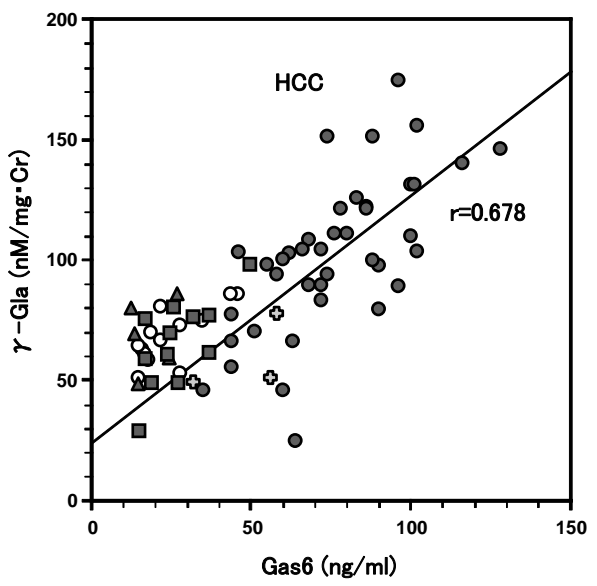

Figure 4. Correlation between Gas6 and $\gamma$-Gla in patients with hepatocellular carcinoma (HCC), acute hepatitis (AH), fulminant hepatitis $(\mathrm{CH})$, chronic hepatitis $(\mathrm{CH})$ and liver cirrhosis (LC). The coefficients of correlation between in HCC $(\bullet: r=0.678, n=40, P<0.05)$, AH $(O: r=0.725, n=$ 13, $\mathrm{P}<0.05), \mathrm{FH}(+\mathrm{r}=0.607, \mathrm{n}=3, \mathrm{P}<0.05), \mathrm{CH}(\Delta: \mathrm{r}=$ $0.230, n=5)$, and LC (n: $r=0.674, n=12, P<0.05)$, respecttively.

The correlation coefficients of Gas 6 and platelet count, and bilirubin in cases with LC were statistically significant, and the correlation coefficients of Gas6 and bilirubin were significant in cases with $\mathrm{FH}$.

\section{Discussion}

The hemostatic proteins are well carboxylated, and their main site of synthesis is liver, which is well vascularized to receive dietary VK absorbed from the gut into blood.

Whether dietary VK is as equally available to other tissue is unknown.

All vitamin $\mathrm{K}$ dependent (VKD) proteins may bind carboxylase through two contact sites, low-affinity and high-affinity, with osteocalcin having reversed affinities to that of the other VKD proteins. Undercarboxylated osteocalcin is frequently observed in the general population, which indicates that incomplete carboxylation occurs in bone where osteocalcin is synthesized. The extent of undercarboxylation is unknown because the analysis is indirect, i.e., on blood samples rather bone tissue [20,21].

The environment of the biosynthesis increase of the Gas6, osteocalcin, and DCP may resemble it [22]. In other words, it is VK-deficiency state and/or serum starvation [21]. Our study demonstrated the increase of Gas6, DCP and urinary $\gamma$-Gla in HCC, however, the precise mechanism(s) of their biosynthesis remain elusive.

On the other hand, the urinary $\gamma$-Gla level in $\mathrm{AH}, \mathrm{CH}$, $\mathrm{FH}$, and LC decreased than the healthy normal controls. In addition, the $\gamma$-Gla level in many cancers increased.

Therefore, it is thought that the VK metabolism for 
these liver diseases is inhibited [22].

A number of recent studies show that increases in Gas6 and its receptor Axl are associated with a poor prognosis for glioblastoma [6] and ovarial carcinoma, [23] and with an improved prognosis for renal cell carcinoma, demonstrating the complexity of the system, [7] and the elevated plasma DCP in HCC patients is associated with a high risk of vascular invasion, intra-hepatic metastasis, tumor recurrence, and DCP is to promote matrix metalloproteinase activity in HCC cell [11].

There studies of Tso, et al. [8] and He, L. et al. about Axl in HCC [9]. A study by He, et al., using cDNA microarray showed that Hca-F cells with higher Axl levels had high lymphatic metastasis potential, and proliferative advantage, and this study also showed that the angiogenic factor protein (Cyr 61) was the principle gene affected by Gas6 stimulation of Axl in the Hca-F cells.

In the liver, Gas6 is mainly expressed in Kupffer cells with levels below those observed in other tissues such as lung, kidney or heart, and the expression of Axl is found in HCC cells [24].

Furthermore, Gas6 was expressed in antigen-presenting cells and endothelial cells (ECs) but not in T-cells. From these evidences, plasma Gas6 originates from ECs and leukocytes (including dendritic cells are macrophages) [25-27].

The present study showed that the plasma Gas6 and sAxl levels were significantly higher in HCC than in the healthy normal adult controls. Furthermore, the Gas6 and sAxl levels were elevated together in a significant correlation in HCC, and those both levels progressively increased with progressing TNM stages in HCC.

Furthermore, the Gas6/sAxl molar ratios in the HCC were significantly higher than in normal adult controls. Gas6 molar level has lower than sAxl in plasma, and the elevated Gas6/sAxl molar ratio in HCC shows relative predominance of Gas6 for TAM system.

As a result, the trend of the increased plasma Gas6 or Gas6/sAxl molar ratio in HCC may reflect the continuance of the cancer proliferation, and those elevated values in the HCC are associated with the poor prognosis. Also, it is thought that the elevation of these values is observed in the inflammation [28].

Gas6 is not only the modulation of cell growth and inhibits apoptosis but also acute-phase reactant, and is increased during sepsis, the associated inflammatory diseases, regulate neovascularization, and rejection of transplants $[27,28]$.

Illustratively, there are many similar reports for renal cell cancer [7], severe sepsis, [28] SLE, [27] and abdominal aortic aneurysms [29].

In these, Gas6 increases in renal cell cancer, severe sepsis, and SLE, but sAxl decreases in abdominal aortic aneurysms.

These ratios showed a positive trend corresponding to the progression of the stage of a disease and may lead to poor prognosis.

A dynamic equilibrium between sAxl and Gas6 levels in plasma may have an important regulatory role and affect Gas6 function. Ekman et al. reported that the measured Kds of recombinant-sAxl for Gas6 binding was 4 fold higher in comparison with that of sAxl in human serum [30].

sAxl may increase the bioavailability of Gas6 by prolonging its half-life and showing ligand release from the complex to provide physiologically relevant levels of Gas6 in tissues, thereby resulting in local or systemic effects of the protein and the nature and/or duration of the signaling event [31].

Recently, Loges et al. provide new evidence on the regulation and significance of Gas6/Axl activity in cancer. Using three different syngeneic models of transplantation of Gas6-/- bone marrow intro wild type mice, they elucidated that tumors educate tumor infiltrating macrophages to secrete high levels of the Gas6 and its Tyro3, Axl, Mer receptors, leading to tumor growth and metastasis. In other words, Gas6 fosters tumor growth by promoting cancer proliferation $[32,33]$.

According to the development of the HCC, DCP having less Gla residues and lost most coagulant ability increases [12,34]. Furthermore, increased plasma DCP in $\mathrm{HCC}$ is related to the presence of vascular invasion, portal vain thrombosis, and intrahepatic metastasis etc. About these, Gas6 exerts prothrombotic properties in the hematopoietic and vascular compartments.

Gas6 that is released (or circulating) subsequently binds to one of the TAMs, which leads to up-regulation of tissue factor. The details of the course are still unknown, and tissue factor can then initiate coagulation, ultimately leading to further thrombotic generation and the formation of fibrin clot [35]. In HCC, the increase of Gas6 may respond to increase of the DCP.

Gas6 secreted by macrophages and hepatic stellate cells (HSC) activated into myofibroblastic cells (HSC/ MFB) after experimental acute and chronic $\mathrm{CCL}_{4}$ injury liver inflammation models promotes HSC and $\mathrm{HSC} / \mathrm{MFB}$ survival and might support transient HSC/MFB accumulation during liver healing $[21,22,36]$.

In another experimental model, the plasma levels of Gas6 increase early after ischemia/reperfusion (I/R), which paralleled the upregulation of Gas6 in hepatic extracts. These findings indicate that Gas6 exerts protective effect against liver I/R injury, emerging as a Gas6 potential novel target in disease clinical setting where hepatic $\mathrm{I} / \mathrm{R}$ damage occurs such as liver transplantation, hemorrhagic shock or liver surgery [36]. 
Therefore, the trend of Gas6 is supplied by the inflammatory process of the liver and normalizes it by a process of the inflammatory healing.

The sAxl, Gas6, and urinary $\gamma$-Gla levels in those liver diseases ( $\mathrm{AH}, \mathrm{FH}, \mathrm{CH}$, and $\mathrm{LC}$ ) show approximately good correlation. As for those facts, it is thought that vitamin $\mathrm{K}$ metabolism is generally inhibited in those liver diseases. However, levels of Gas6 that not produced with hepatocyte are always lower than levels of sAxl in those liver diseases and normal controls. Therefore, the Gas6/sAxl molar ratios in liver diseases $(\mathrm{AH}, \mathrm{FH}, \mathrm{CH}$, LC) decreased significantly in comparison with those $\mathrm{HCC}$, and healthy normal controls.

FH did not lead to healing, but the Gas6/sAxl molar ratios were lower than normal adult controls. As for this, various functional deficiency of Gas6 may have brought poor prognosis of $\mathrm{FH}$. Furthermore, the mechanism of the decrease of the secretion of Gas6 in FH may be inhibition of the VK metabolism, increase of cytokines/chemokines or any role for Mer. Future examination is necessary about these.

High sAxl levels may prevent Gas6 interaction with the membrane-bound receptors and inhibit Gas6 specific signaling, whereas low sAxl levels could potentiate Gas6 activity, but further examinations are necessary so that those facts are proved.

A potential ability of sAxl to serve as a natural antagonist of Gas6 could have clinical relevance.

Therefore, we thought that Gas6 and sAxl and Gas6/ sAxl ratio in progress $\mathrm{HCC}$ increased than other liver diseases (AH, FH, CH, and LC). These measurements suggest possibility as the biomarkers of the HCC therapy.

When we considered potential treatment for cancer by conventional results, at first a strategy to block supply of Gas6 for the cancer cell is assumed, and a strategy to inhibition Axl signaling pathway for cancer cell is assumed.

Further studies are needed to establish Gas6 and sAxl as important biomarkers for correlation with disease stage and predicting prognosis.

\section{REFERENCES}

[1] G. Manfioletti, C. Brancolini, G. Avnzi and C. Schneider, "The Protein Encoded by a Growth Arrest-Specific Gene (gas6) Is a New Member of the Vitamin K-Dependent Proteins Related to Protein S, a Negative Coregulator in the Blood Coagulation Cascade," Molecular and Cellular Biology, Vol. 13, No. 8, 1993, pp. 4976-4958.

[2] K. Nagata, K. Ohash, T. Nakano, et al., "Identification of the Product of Growth Arrest-Specific Gene 6 as a Common Ligand for Axl, Sky, and Mer Receptor Tyrosine Kinases," The Journal of Biological Chemistry, Vol. 271, 1996, pp. 3022-3027.

\section{doi:10.1074/jbc.271.47.30022}

[3] P. Marcandalli, M. Gostissa, B. Varnum, S. Goruppi and C. Schneider, "Identification and Tissue Expression of Splice Variant for the Growth Aresst-Specific Gene Gas6," FEBS Letters, Vol. 415, No. 1, 1997, pp. 56-58. doi:10.1016/S0014-5793(97)01094-6

[4] I. Hasanbasic, J. Cuerquis, B. Varnum and D. M. Blostein, "Intercellular Signaling Pathways Involved in Gas6-AxlMediated Survival of Endothelial Cells," American Journal of Physiology-Heart and Circulatory Physiology, Vol. 287, No. 3, 2004, pp. H1207-1213.

[5] R. M. Linger, A. K. Keating, H. S. Earp and D. K. Graham, "TAM Receptor Tyrosine Kinases: Biologic Functions, Signaling and Potential Therapeutic Targeting in Human Cancer," Advances in Cancer Research, Vol. 100, 2008, pp. 35-83.

[6] M. Hutterer, P. Knyazev, A. Abate, et al., "Axl and Growth Arrest-Specific Gene 6 Are Frequently Overexpressed in Human Gliomas and Predict Poor Prognosis in Patients with Glioblastoma Multiforme," Clinical Cancer Research, Vol. 14, No. 1, 2008, pp. 130-138.

[7] A. Gustafsson, D. Martuszewska, M. Johansson, et al., "Differential Expression of Axl and Gas6 in Renal Cell Carcinoma Reflecting Tumor Advancement and Survival," Clinical Cancer Research, Vol. 15, No. 14, 2009 , pp. 4742-4749.

[8] A. P. Tsou, K. M. Wu, T. Y. Tsen, et al., "Parallel Hybridization Analysis of Multiple Protein Kinase Genes: Identification of Gene Expression Patterns Characteristic of Human Hepatocellular Carcinoma," Genomics, Vol. 50, No. 3, 2010, pp. 331-340.

[9] L. He, J. Zhang, L. Jiang, et al., "Differential Expression of Axl in Hepatocellular Carcinoma and Correlation with Tumor Lymphatic Metastasis," Molecular Carcinogenesis, Vol. 49, No. 10, 2010, pp. 882-891. doi: $10.1002 / \mathrm{mc} .20664$

[10] V. Budagian, E. Bulanova, Z. Orinska, et al., "Soluble Axl Is Generated by ADAM10-Dependent Cleavage and Associates with Gas6 in Mouse Serum," Molecular and Cellular Biology, Vol. 25, No. 21, 2005, pp. 9324-9339. doi:10.1128/MCB.25.21.9324-9339.2005

[11] B. Furie, B. A. Bouchard and B. C. Furie, "Vitamin K-Dependent Biosynthesis of Gamma-Carboxyglutamic Acid," Blood, Vol. 93, No. 6, 1999, pp. 1798-1808.

[12] Y. Inagaki, W. Tang, H. Xu, et al., "Des- $\gamma$-Carboxyprothrombin: Clinical Effectiveness and Biochemical Importance," BioScience Trends, Vol. 2, No. 2, 2008, pp. 5360.

[13] P. Yue, Z. H. Gao, X. Xue, et al., "Des-Ganmma-Carboxy Prothrombin Induces Matrix Metalloproteinase Activity in Hepatocellular Carcinoma Cells by Involving the ERK1/2 MARPK Signaling Pathway," European Journal of Cancer, Vol. 47, No. 7, 2011, pp. 1115-1124. doi:10.1016/j.ejca.2011.01.017

[14] N. Hotta, M. Avada, K. Sato, et al., "Effect of Vitamin $\mathrm{K} 2$ on the Recurrence in Patients with Hepatocellular Carcinoma," Hepatogastroentrology, Vol. 54, No. 79, 2007, pp. 2073-2077. 
[15] H. Yoshida, Y. Shratori, M. Kudo, et al., "Effect of Vitamin K2 on the Recurrence of Heptocellular Carcinoma," Hepatology, Vol. 54, No. 2, 2011, pp. 532-540. doi: $10.1002 /$ hep. 24430

[16] Liver Cancer Study Group of Japan, "The General Rules for the Clinical and Pathological Study of Primary Liver Cancer," 4th Edition, Kanehara, Tokyo, 2000.

[17] M. Kudo, H. Chung and Y. Osaki, "Prognostic Staging System for Hepatocellular Carcinoma (CLIP Score): Its Value and Limitations, and a Proposal for a New Staging System, the Japan Integrated Staging Score (JIS Score)," Journal of Gastroenterology, Vol. 38, No. 3, 2003, pp. 207-215. doi:10.1007/s005350300038

[18] A. Fujiwara, S. Mochida, A. Matsui, N. Nakayama, S. Nagoshi and G. Toda, "Intractable Liver Diseases Study Group Of Japan Fulminant Hepatitis and Late Onset Hepatic Failure in Japan," Hepatology Research, Vol. 38, No. 7, 2008, pp. 646-657. doi:10.1111/j.1872-034X.2008.00322.x

[19] M. Kuwada and K. Katayama, "An Improved Method for Determination of $\gamma$-Carboxyglutamic Acid in Proteins, Bone and Urine," Analytical Biochemistry, Vol. 131, No. 1, 1983, pp. 173-179. doi:10.1016/0003-2697(83)90150-1

[20] K. L. Berkner, "The Vitamin K-Dependent Carboxylase," Annual Review of Nutrition, Vol. 25, 2005, pp. 127-149. doi:10.1146/annurev.nutr.25.050304.092713

[21] J. C. McCann, B. N. Ames and K. Vitamin, “An Example of Triage Theory: Is Micronutrient Inadequacy Linked to Disease of Aging?" The American Journal of Clinical Nutrition, Vol. 90, No. 4, 2009, pp. 889-907. doi:10.3945/ajen.2009.27930

[22] K. Gotoh, S. Kato, S. Sumiya, S. Uehara, et al., "Urinary Levels of $\gamma$-Carboxyglutamic Acid and Its Clinical Significance," Biological \& Pharmaceutical Bulletin, Vol. 17, No. 1, 1994, pp. 142-145. doi:10.1248/bpb.17.142

[23] W. Sun, J. Fujimoto and T. Tamaya, "Coexpression of Gas6/ Axl in Human Ovarian Cancers," Oncology, Vol. 66, No. 6, 2004, pp. 450-457. doi:10.1159/000079499

[24] D. Couchie, F. Lafdil, N. Martin-Garcia, Y. Laperche, E. S. Zafrani and P. Mavier "Expression and Role of Gas6 Protein and of Its Receptor Axl in Hepatic Regeneration from Oval Cells in the Rat," Gastroenterology, Vol. 129, No. 5, 2005, pp. 1633-1642. doi:10.1053/j.gastro.2005.08.004

[25] F. Lafdil, M. N. Chobert, D. Couchie, et al., "Induction of Gas6 Protein in CCL4-Induced Rat Liver and Antiapoptotic Effect on Hepatic Stellate Cells," Hepatology, Vol. 44, No. 1, 2006, pp. 228-239. doi:10.1002/hep.21237

[26] F. Lafdil, M. N. Chobert, V. Deveaux, et al., "Growth Arrest-Specific Protein 6 Deficiency Impairs Liver Tissue Repair after Acute Toxic Hepatitis in Mice," Journal of
Hepatology, Vol. 51, No. 1, 2009, pp. 55-66.

doi:10.1016/j.jhep.2009.02.030

[27] M. Tjiwa, L. Bellido-Martin, Y. Lin, et al., "Gas6 Promotes Inflammation by Enhancing Interactions between Endothelial Cells, Platelets, and Leukocytes," Blood, Vol. 111, No. 8, 2008, pp. 4096-4105. doi:10.1182/blood-2007-05-089565

[28] C. Ekman, A. Jönsen, G. Sturfelt, A. A. Bengtsson and B. Dahlbäck, "Plasma Concentrations of Gas6 and sAxl Correlate with Disease Activity in Systemic Liphus Erythematosus and Its Soluble Tyrosine Kinase Receptor sAxl in Sepsis and Systemic Inflammatory Response Syndromes," Critical Care, Vol. 14, No. 4, 2010, p. R158.

[29] C. Ekman, D. F. Site, A. Gottsäter, B. Lindblad and B. Dahlbäck, "Plasma Concentrations of Growth Arrest Specific Protein 6 and the Soluble Form of Its Tyrosine Kinase Receptor Axl as Markers of Large Abdominal Aortic Aneurysms," Clinical Biochemistry, Vol. 43, No. 1-2, 2010, pp. 110-114. doi:10.1016/j.clinbiochem.2009.07.025

[30] C. Ekman, J. Stenhoff and B. Dahlbäck, "Gas6 Is Complexed to Souble Tyrosine Kinase Receptor Axl in Human Blood," Journal of Thrombosis and Haemostasis, Vol. 8, No. 4, 2010, pp. 838-844.

[31] A. Verma, S. L. Wamer, H. Vankayalapati, D. J. Bearss and S. Shrma, "Targeting Axl and Mer Kinases in Cancer," Molecular Cancer Therapeutics, Vol. 10, No. 10, 2011, pp. 1763-1773. doi:10.1158/1535-7163.MCT-11-0116

[32] L. Loges, T. Schmidt, M. Tjwa, et al., "Malignant Cells Fuel Tumor Growth by Educating Infilutrating Leukocytes to Produce the Mitogen Gas6," Blood, Vol. 115, No. 11, 2010, pp. 2264-2273. doi:10.1182/blood-2009-06-228684

[33] A. Sica, "Macrophages Gives Gas6 to Cancer," Blood, Vol. 115, No. 11, 2010, pp. 2122-2123. doi:10.1182/blood-2009-12-255869

[34] S. Uehara, K. Gotoh, H. Handa, H. Tomita and M. Senshuu, "Distribution of the Heterogeneity of Des- $\gamma$-Carboxyprothrombin in Patients with Hepatocellular Carcinoma," Journal of Gastroenterology and Hepatology, Vol. 20, No. 10, 2005, pp. 1545-1552. doi:10.1111/j.1440-1746.2005.03899.x

[35] J. H. Foley and E. M. Conway, "Gas6 Gains Entry into the Coagulation Cascade," Blood, Vol. 121, No. 4, 2013, pp. 570-571. doi:10.1182/blood-2012-11-468678

[36] L. Licuna, C. Bárcena, L. Bellido-Martin, et al., "Growth Arrest-Specific Protein 6 Is Hepatoprotective against Ischemia/Reperfusion Injury," Hepatology, Vol. 52, No. 4, 2010, pp. 1371-1379. 